\title{
七、八元瓜环与两种含香豆素单元的紫精衍生物的相互作用
}

\author{
崔艳婷赵广宽王瑞欣朱卫国* 邹大鹏* \\ (郑州大学化学与分子工程学院 河南省化学生物与有机化学重点实验室 郑州 450052)
}

\begin{abstract}
摘要 合成了两个含有香豆素单元的紫精衍生物(简称 [XDS-3CMV], [XDS-6CMV]). 利用 ${ }^{1} \mathrm{H}$ NMR, ESI-MS, UV-Vis 以 及苂光光谱分析方法考察了七八元瓜环 $\mathrm{CB}[n](n=7,8)$ 与客体分子 [XDS-3CMV], [XDS-6CMV]之间的相互作用. 结果 显示, 两种客体均可与 $\mathrm{CB}[n](n=7,8)$ 分别形成稳定的包结配合物, 烷基链长度以及瓜环空腔尺寸的差异影响主客体 相互作用的模式. 其中七元瓜环与两种客体分子的紫精部分作用形成 $1: 1$ 的包结配合物; 八元瓜环可以与折叠成 U 型 结构的 [XDS-6CMV]分子形成 $1: 1$ 的包结物, 而与[XDS-3CMV]分子则形成超分子聚合物.
\end{abstract}

关键词 $\mathrm{CB}[n](n=7,8)$; 紫精; 香豆素; 包结配合物

\section{Interaction between Cucurbit[n]uril $(n=7,8)$ and Two Kinds of Viologen Derivatives Containing Coumarin Unit}

\author{
Cui, Yanting Zhao, Guangkuan Wang, Ruixin Zhu, Weiguo* Zou, Dapeng* \\ (Key Laboratory of Chemical Biology and Organic Chemistry of Henan Province, College of Chemistry and \\ Molecular Engineering, Zhengzhou University, Zhengzhou 450052)
}

\begin{abstract}
Two kinds of viologen derivatives ([XDS-3CMV] and [XDS-6CMV]) containing coumarin unit were synthesized and characterized. Spectroscopic techniques such as ${ }^{1} \mathrm{H}$ NMR, ESI-MS, UV-Vis and fluorescence spectroscopies were used to investigate the interaction between $\mathrm{CB}[n](n=7,8)$ and guest molecules. The results reveal that each of the two guest binds with cucurbit $[n]$ uril $(n=7,8)$ to form stable inclusion complexes. The length of the alkyl chain of the guests and the size of cucurbit[ $n]$ uril $(n=7,8)$ can affect the binding models of the inclusion complexes. Cucurbit[7]uril primarily binds with the viologen unit of the two guest molecules to form $1: 1$ inclusion complexes. [XDS-6CMV] prefers to fold back to form a $1: 1$ U-shaped complex with cucurbit[8]uril. Whereas [XDS-3CMV] prefers to form supramolecular polymer with cucurbit[8]uril.

Keywords cucurbit $[n]$ uril $(n=7,8)$; viologens; coumarin; inclusion complexes
\end{abstract}

瓜环 ${ }^{[1]}$ (cucurbit $[n]$ uril, $n=5 \sim 8,10$ ), 又记作 $\mathrm{CB}[n]$ $(n=5 \sim 8,10)$, 是一类新型的高度对称的笼状大环超分 子主体化合物(如 Scheme 1), 因其自身特殊的腔体结构 以及良好的分子识别性能, 在超分子家族中占据了日趋 重要的位置, 尤其是在主客体化学和分子识别方面的应 用被广泛研究 ${ }^{[2,3]}$. 研究发现七元瓜环(CB[7])可以与紫 精、二茂铁及过渡金属类化合物等自组装形成 $1: 1$ 的 主客体配合物 ${ }^{[4 \sim 8]}$. 罗全课题组 ${ }^{[9]}$ 研究了客体分子和 $\mathrm{CB}[7]$ 组成的分子胶囊的自组装和解离过程. 八元瓜环 $(\mathrm{CB}[8])$ 具有比七元瓜环更大的端口和空腔, 因此可以 和紫精、哌嗪以及弯曲的烷基链等包结形成更加复杂的
二元或者三元包结配合物 ${ }^{[10 ~ 14]}$, 孙世国课题组 ${ }^{[15]}$ 研究 发现苯-紫精类化合物和 $\mathrm{CB}[8]$ 可形成 $1: 1$ 的二元包合 物. 紫精衍生物作为一种典型的缺电子体, 可以和瓜环 形成良好的包结物. Kaifer ${ }^{[16]}, \mathrm{Kim}^{[17]}$ 及孙立成课题组 ${ }^{[18]}$ 都有这方面的研究. 我们课题组 ${ }^{[19]}$ 研究发现紫精衍生 物的烷基链部分可以与对苯二酚和紫精部分组成的电 荷转移对一起进入八元瓜环空腔内部. 谭业邦课题 组 ${ }^{[20]}$ 研究后发现客体苂光分子可以和甲基紫精一起进 入 $\mathrm{CB}[8]$ 空腔形成 $1: 1: 1$ 的包结配合物. 孙立成课题 组 ${ }^{[21]}$ 研究发现 $\mathrm{CB}[8]$ 可以和由三联吡啶、紫精单元和荟 酚单元组成的化合物形成 $1: 1$ 的包结物, 紫精单元和

\footnotetext{
*E-mail: zdp@zzu.edu.cn

Received November 13, 2013; revised January 2, 2014; published online February 12, 2014.

Project supported by the Basic and Frontier Technology Research Program of Henan Province (No. 122300413203).

河南省基础与前沿技术研究计划(No. 122300413203)资助项目.
} 
菜酚单元折叠后共同进入 $\mathrm{CB}[8]$ 空腔. 陈传峰课题组 ${ }^{[22}$ ${ }^{24]}$ 设计合成了三蝶烯类大环主体化合物和一系列紫精 衍生物, 研究发现该主体化合物和部分客体分子在浓度 较低时形成 $1: 1$ 配合物, 浓度较大或液态时可形成阶 梯状超分子聚合物. 香豆素 ${ }^{[24]}$ 是广泛存在植物中的内 酯类杂环有机化合物, 具有较好的光学活性和良好的生 物活性, 被广泛地应用于制药、荧光材料以及分析识别 等领域 ${ }^{[25 ~ 27]}$. 我们设计合成了两种水溶性良好的含香 豆素单元的紫精衍生物 [XDS-6CMV] 和 [XDS-3CMV] (Scheme 1), 并通过 ${ }^{1} \mathrm{H}$ NMR, ESI-MS, UV-Vis 和苂光光 谱法研究了其与 $\mathrm{CB}[7], \mathrm{CB}[8]$ 之间的相互作用, 结果表 明这两个客体与 $\mathrm{CB}[7], \mathrm{CB}[8]$ 均能形成稳定的包结配合 物, 但包结模式存在显著差异. 我们的研究结果将为超 分子主体化合物瓜环与香豆素类衍生物在药物学、生物 学、光学材料以及环境科学等方面的综合研究和应用提 供更加广泛的理论基础.

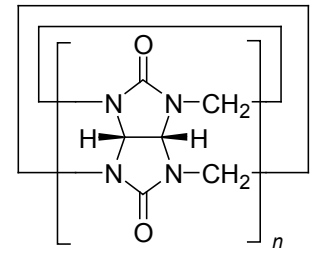

Cucurbit $[n]$ uril $(n=7,8)$

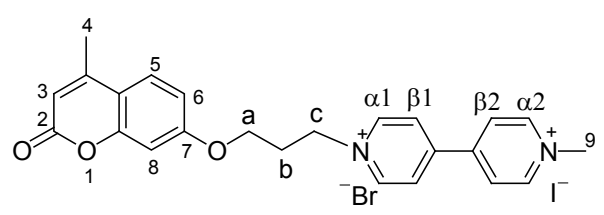

[XDS-3CMV]

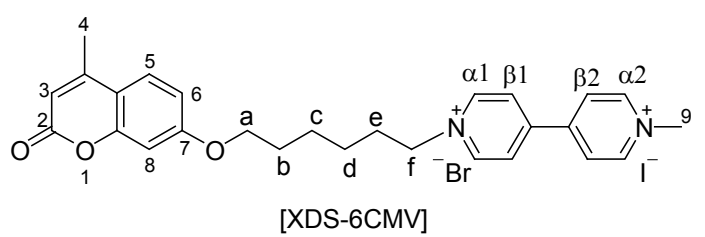

Scheme 1

\section{1 结果与讨论}

\section{$1.1{ }^{1} \mathrm{H}$ NMR 图谱解析}

1.1.1 [XDS-6CMV]与 $\mathrm{CB}[n](n=7,8)$ 相互作用的 ${ }^{1} \mathrm{H}$ NMR 图谱解析

核磁共振技术是研究瓜环和客体分子化合物包结 过程的有效手段. 图 1 为摩尔比法测定的 [XDS-6CMV] 与 $\mathrm{CB}[7]$ 相互作用的核磁氢谱. 当加入 0.5 equiv. 的 $\mathrm{CB}[7]$ 时, 核磁图 1B 中既有游离的客体的质子峰, 又有 [XDS-6CMV] 与 $\mathrm{CB}[7]$ 形成的配合物的质子峰, 说明客 体与瓜环作用与脱离的交换频率小于仪器的工作频率.
当加入 1.0 equiv.的 $\mathrm{CB}$ [7]时, 图 $1 \mathrm{C}$ 只观察到配合物对 应的质子峰，与游离的[XDS-6CMV]图 1A 相比，紫精基 才上的 $\mathrm{H}(\alpha 1), \mathrm{H}(\alpha 2)$ 分别向高场移动了 $\delta 0.17,0.10$, $\mathrm{H}(\beta 1), \mathrm{H}(\beta 2)$ 均向高场移动了 $\delta 1.32$, 与紫精相连的 $\mathrm{H}(9)$ 向低场移动了 $\delta 0.11$, 说明紫精部分进入了瓜环空腔受 到了瓜环的屏蔽效应的影响，而紫精上的甲基处在瓜环 端口外部. 香豆素部分的质子化学位移变化不是很明 显, $\mathrm{H}(3), \mathrm{H}(4), \mathrm{H}(5), \mathrm{H}(6), \mathrm{H}(8)$ 分别向低场移动了 $\delta 0.03$, $0.11,0.03,0.06,0.20$, 说明这些质子氢都处于瓜环外部, 而且离瓜环较远，所以受瓜环的影响比较小. 烷基链上 的质子氢 $\mathrm{H}(\mathrm{a}), \mathrm{H}(\mathrm{b}), \mathrm{H}(\mathrm{c}), \mathrm{H}(\mathrm{d}), \mathrm{H}(\mathrm{e})$ 均移向低场, 只有 $\mathrm{H}(\mathrm{f})$ 向高场移动, 说明离紫精单元较近的亚甲基上的 $\mathrm{H}(\mathrm{f})$ 进入空腔内, 其余的烷基链以及香豆素部分均在瓜 环空腔外部. 当加入过量的 $\mathrm{CB}[7]$ 后, 核磁图谱几乎没 有变化. 由此我们推断[XDS-6CMV] 与 $\mathrm{CB}$ [7]以 $1: 1$ 的 包结比形成配合物，紫精基团和连接紫精单元的第一个 亚甲基进入瓜环疏水性空腔内部(Scheme 2).

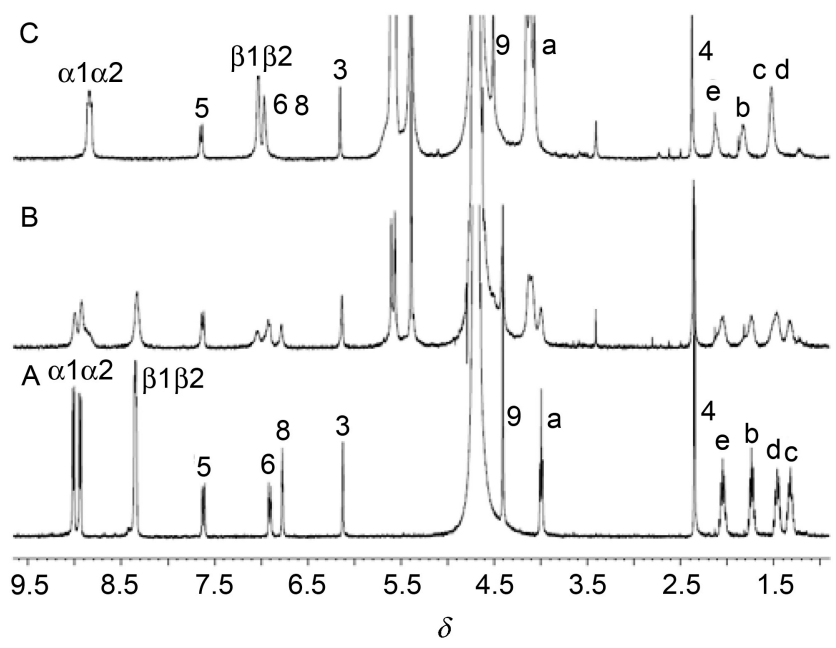

图 1 [XDS-6CMV] 与 $\mathrm{CB}[7]$ 相互作用的 ${ }^{1} \mathrm{H}$ NMR 图谱(400 $\left.\mathrm{MHz}, \mathrm{D}_{2} \mathrm{O}\right)$

Figure $1{ }^{1} \mathrm{H}$ NMR spectra of interaction between $\mathrm{CB}$ [7] and [ XDS-6CMV] (400 MHz, $\left.\mathrm{D}_{2} \mathrm{O}\right)$

(A) $[\mathrm{XDS}-6 \mathrm{CMV}]$, (B) $n(\mathrm{CB}[7]) / n([\mathrm{XDS}-6 \mathrm{CMV}])=0.5$, (C) $n(\mathrm{CB}[7]) /$ $n([\mathrm{XDS}-6 \mathrm{CMV}])=1.0$

图 2 为摩尔比法测定的 $[\mathrm{XDS}-6 \mathrm{CMV}$ 与 $\mathrm{CB}[8]$ 相互 作用的核磁氢谱. 当加入 0.5 equiv. 的 $\mathrm{CB}$ [8]时, 图 2B 中 同时存在游离客体和配合物的质子峰。当加入 1.0 equiv. 的 $\mathrm{CB}$ [8]时, 图 $2 \mathrm{C}$ 中只能观察到包结物的质子峰. 与图 $2 \mathrm{~A}$ 相比较[XDS-6CMV]中所有质子氢的化学位移 均向高场发生了不同程度的移动, 其中紫精上的 $H(\alpha 1)$, $\mathrm{H}(\alpha 2), \mathrm{H}(\beta 1), \mathrm{H}(\beta 2)$, 分别向高场移动了 $\delta 0.38,0.77$, $0.66,0.87$, 香豆素单元上的 $\mathrm{H}(3), \mathrm{H}(4), \mathrm{H}(5), \mathrm{H}(6), \mathrm{H}(8)$ 分别向高场移动了 $\delta 0.36,0.28,0.43,0.73,0.59$, 烷基链 上的 $\mathrm{H}(\mathrm{a}), \mathrm{H}(\mathrm{b}), \mathrm{H}(\mathrm{c}), \mathrm{H}(\mathrm{d}), \mathrm{H}(\mathrm{e}), \mathrm{H}(\mathrm{f})$ 均向高场移动, 


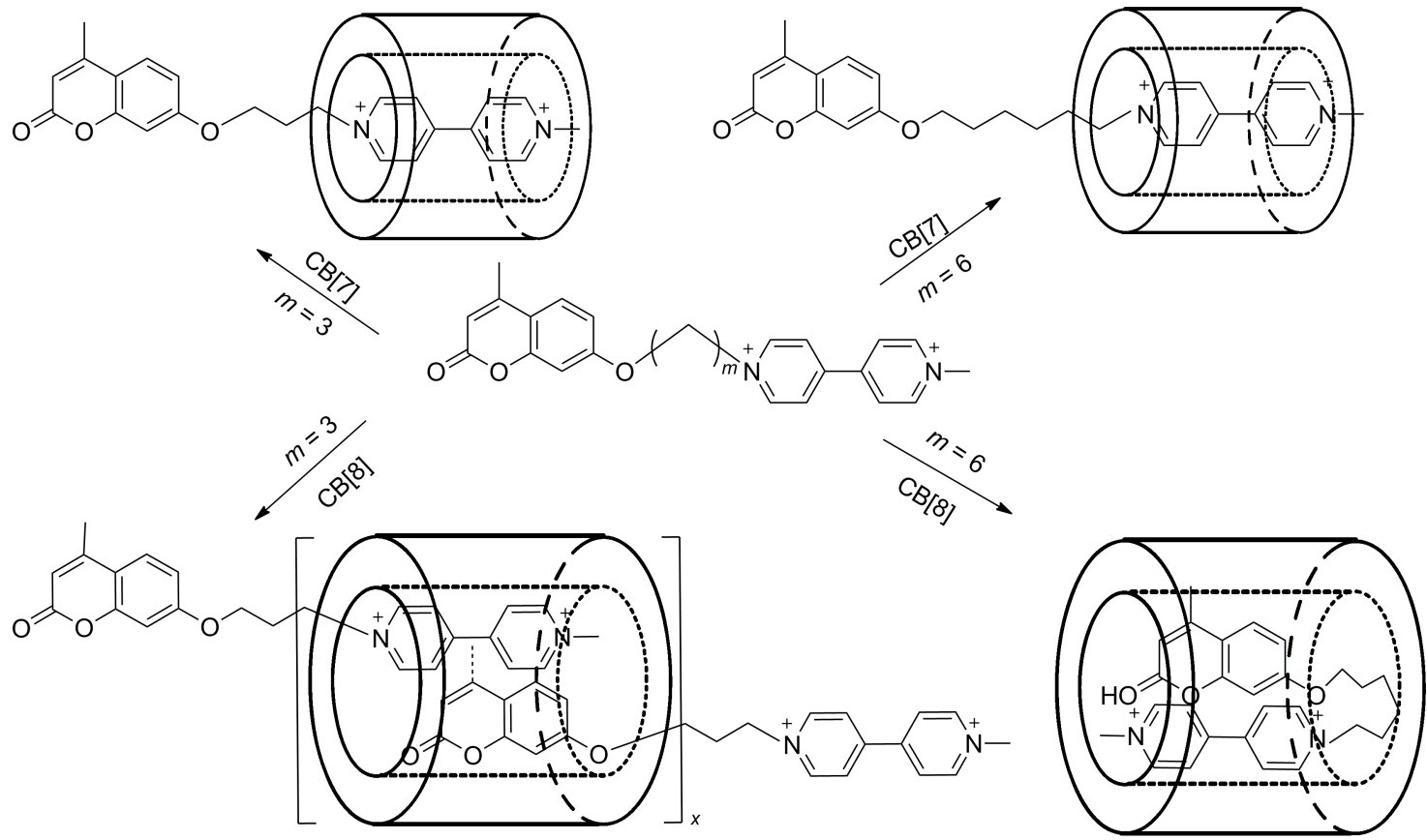

Scheme 2

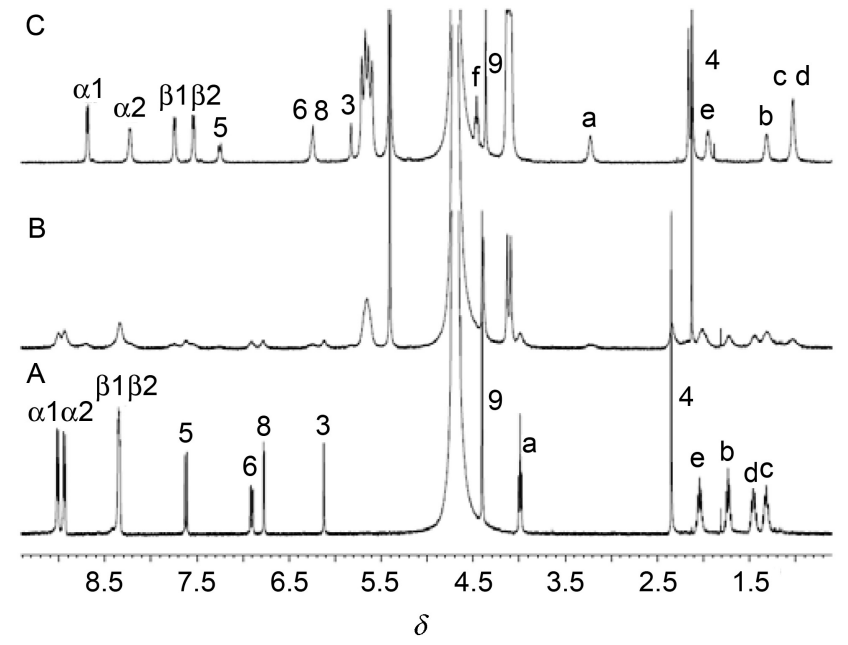

图 2 [XDS-6CMV]与 $\mathrm{CB}[8]$ 相互作用的 ${ }^{1} \mathrm{H}$ NMR 图谱(400 $\left.\mathrm{MHz}, \mathrm{D}_{2} \mathrm{O}\right)$

Figure $2{ }^{1} \mathrm{H}$ NMR spectra of interaction between $\mathrm{CB}[8]$ and [ XDS-6CMV] $\left(400 \mathrm{MHz}, \mathrm{D}_{2} \mathrm{O}\right)$

(A) $[\mathrm{XDS}-6 \mathrm{CMV}],(\mathrm{B}) n(\mathrm{CB}[8]) / n([\mathrm{XDS}-6 \mathrm{CMV}])=0.5$, (C) $n(\mathrm{CB}[8]) /$ $n([\mathrm{XDS}-6 \mathrm{CMV}])=1.0$

$\mathrm{CB}[8]$ 与 $[\mathrm{XDS}-6 \mathrm{CMV}]$ 的摩尔比大于 $1: 1$ 后, 核磁图谱 没有变化. 据此我们推断[XDS-6CMV] 与 $\mathrm{CB}[8]$ 形成了 $1: 1$ 的包结物, 此时整个客体分子可能采取 $\mathrm{U}$ 型的折 叠型结构全部处于八元瓜环空腔内部(Scheme 2).

1.1.2 [XDS-3CMV]与 $\mathrm{CB}[n](n=7,8)$ 相互作用 ${ }^{1} \mathrm{H}$ NMR 图谱解析

为了研究烷基链长度对超分子包结体系的影响, 设 计合成了另一种目标化合物 [XDS-3CMV]. 图 3 为
[XDS-3CMV] 与 $\mathrm{CB}[7]$ 相互作用的 ${ }^{1} \mathrm{H}$ NMR 谱图. 当加 入 0.5 equiv. 的 $\mathrm{CB}[7]$ 时, 图 3B 中可以同时观察到游离 的 [XDS-3CMV]及包结配合物的质子峰; 当加入 1.0 equiv. 的 $\mathrm{CB}[7]$ 时, 图 3C 中只观察到包结物的质子峰. 与图 $3 \mathrm{~A}$ 相比, 紫精单元上的 $\mathrm{H}(\beta 1), \mathrm{H}(\beta 2)$ 均向高场移动 了 $\delta 2.04, \mathrm{H}(\alpha 1), \mathrm{H}(\alpha 2)$ 也向高场分别移动了 $\delta 0.11,0.13$. 与紫精单元相连的甲基的质子向低场移动了 $\delta 0.05$. 这 说明紫精单元进入了七元瓜环的空腔，而紫精上的甲基 位于空腔外部. 香豆素单元的质子 $\mathrm{H}(3), \mathrm{H}(4), \mathrm{H}(5)$, $\mathrm{H}(6), \mathrm{H}(8)$ 分别向低场移动了 $\delta 0.03,0.11,0.10,0.33$, 0.41 , 而烷基链上的质子均向低场移动, 说明这些质子 位于瓜环端口之外. 当加入大于 10 equiv.的 $\mathrm{CB}$ [7]时, 其核磁图谱与图 3C 几乎一致. 由此可推断 [XDS$3 \mathrm{CMV}$ 与 $\mathrm{CB}$ [7]的包结比为 $1: 1$. 与[XDS-6CMV]和 $\mathrm{CB}[7]$ 的包结模式所不同的是, [XDS-3CMV]和 $\mathrm{CB}[7]$ 包 结时连接紫精部分的亚甲基没有进入瓜环空腔，说明烷 基链的长度对包结模式有一定的影响(Scheme 2).

当 $\mathrm{CB}[8]$ 加入到 $[X D S-3 \mathrm{CMV}$ 氝代水溶液后, 溶液 中出现了大量的黄色沉淀, 静置后的 ${ }^{1} \mathrm{H} N \mathrm{NR}$ 吸收峰很 弱而且为包状. 可能是由于 $\mathrm{CB}[8]$ 加入后, 与 XDS$3 \mathrm{CMV}$ ]形成了稳定的溶解度较小的超分子聚合物从水 中沉淀出来, 所以从 ${ }^{1} \mathrm{H}$ NMR 谱图中无法了解其包结模 式, 需要借助 ESI-MS, UV-Vis 以及苂光发射光谱来进 行综合分析. 这个现象与[XDS-6CMV] 和 $\mathrm{CB}$ [8]的相互 作用得到的核磁图谱图 1 相比较, 是有很大的差别的, 由于客体分子烷基链的长度的差异导致了其性质的差 


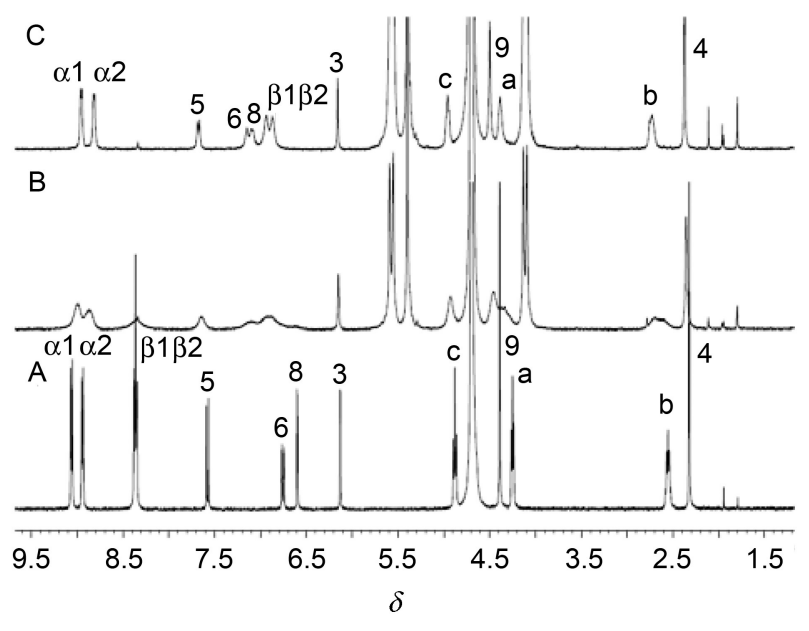

图 3 [ XDS-3CMV] 与 $\mathrm{CB}[7]$ 相互作用的 ${ }^{1} \mathrm{H}$ NMR 图谱(400 $\left.\mathrm{MHz}, \mathrm{D}_{2} \mathrm{O}\right)$

Figure $3{ }^{1} \mathrm{H}$ NMR spectra of interaction between $\mathrm{CB}[7]$ and [ XDS-3CMV] $\left(400 \mathrm{MHz}, \mathrm{D}_{2} \mathrm{O}\right)$

(A) [XDS-3CMV], (B) $n(\mathrm{CB}[7]) / n([\mathrm{XDS}-3 \mathrm{CMV}])=0.5$, (C) $n$ (CB[7]) $/$ $n([\mathrm{XDS}-3 \mathrm{CMV}])=1.0$

异在这里有所体现.

\subsection{ESI-MS 解析}

1.2.1 [XDS-6CMV]与 $\mathrm{CB}[n](n=7,8)$ 相互作用的 ESI-MS 图谱解析

将[XDS-6CMV] 与 $\mathrm{CB}[7], \mathrm{CB}[8]$ 按物质的量比为 $1: 2$ 配成水溶液进行 ESI-MS 测定, 得到 $m / z=796.7$ 的 峰(计算值: $\left[\mathrm{XDS}-6 \mathrm{CMV}+\mathrm{CB}[7]-\mathrm{Br}^{-}-\mathrm{I}^{-}\right]^{2+}, 796.3$ )及 $m / z=879.9$ 的峰(计算值: XDS-6CMV $+\mathrm{CB}[8]-\mathrm{Br}^{-}-$ $\left.\left.\mathrm{I}^{-}\right)^{2+}, 879.3\right)$. 说明 $\mathrm{CB}$ [7], $\mathrm{CB}[8]$ 与 [XDS-6CMV]均按照 $1: 1$ 的包结比形成了稳定的包结配合物. 实验结果更 进一步验证了核磁分析的结果.

1.2.2 [XDS-3CMV] 与 $\mathrm{CB}[n](n=7,8)$ 相互作用的 ESI-MS 图谱解析

同样条件下分别测定[XDS-3CMV]与 $\mathrm{CB}[7], \mathrm{CB}[8]$ 的包结物, 得到 $\mathrm{m} / \mathrm{z}=775.7$ 的峰(计算值: [XDS$\left.\left.3 \mathrm{CMV}+\mathrm{CB}[7]-\mathrm{Br}^{-}-\mathrm{I}^{-}\right]^{2+}, 775.3\right)$ 及 $m / z=858.8$ 的峰 (计算值: [XDS-6CMV $\left.+\mathrm{CB}[8]-\mathrm{Br}^{-}-\mathrm{I}^{-}\right]^{2+}, 858.3$ ). ESI-MS 结果表明 CB[7], CB[8]与[XDS-3CMV]也是按照 $1: 1$ 的包结比形成了稳定的包结配合物. $\mathrm{CB}[8]$ 与 [XDS-3CMV] 的包结体系中也能测到 $1: 1$ 的包结物, 可 能是由于质谱的待测样品浓度比核磁待测样品较低, 而 且 ESI-MS 的灵敏度较高, 能够捕捉到水溶液中微量的 $1: 1$ 包结配合物的信息.

\subsection{UV-Vis 图谱解析}

1.3.1 [XDS-6CMV]与 $\mathrm{CB}[n](n=7,8)$ 相互作用的 UV-Vis 图谱解析

瓜环在大于 $210 \mathrm{~nm}$ 的区域没有特征吸收, 而
[XDS-6CMV] 在水溶液中的吸收峰在 $260,320 \mathrm{~nm}$ 处，其 中 $260 \mathrm{~nm}$ 为紫精的特征吸收峰, $320 \mathrm{~nm}$ 处为香豆素基 团的特征吸收峰. 图 4A, 4B 分别为摩尔比法测定的 [XDS-6CMV] 与 $\mathrm{CB}[7], \mathrm{CB}[8]$ 相互作用的紫外图谱. 从 图 4A 中我们可以观察到, 随着 $\mathrm{CB}[7]$ 浓度的增加, 260 $\mathrm{nm}$ 处的紫精基团的吸收波长略有红移，吸光度也呈现 减弱趋势. 这表明只有紫精部分进入了 $\mathrm{CB}[7]$ 的疏水空 腔内部. 从图 4B 中可以看到, 随着 $\mathrm{CB}[8]$ 浓度的增加, $260,320 \mathrm{~nm}$ 处的紫外吸收峰都发生了明显的红移，吸 光度也呈现规律性的递减趋势. 说明香豆素单元和紫精 单元都受到了瓜环的影响, 可能是由于整个分子都进入 了 $\mathrm{CB}[8]$ 的空腔内部，从而影响了整个分子体系的结构 和电子分布，导致紫外吸收发生明显变化，这也进一步 验证了核磁图谱分析的结果. 根据 Hildebrand-Benesi 方 程计算得到 XDS-6CMV 与 $\mathrm{CB}[7], \mathrm{CB}[8]$ 的包结稳定常 数分别为 $(2.17 \pm 0.40) \times 10^{4},(7.17 \pm 0.36) \times 10^{4} \mathrm{~L} / \mathrm{mol}$.
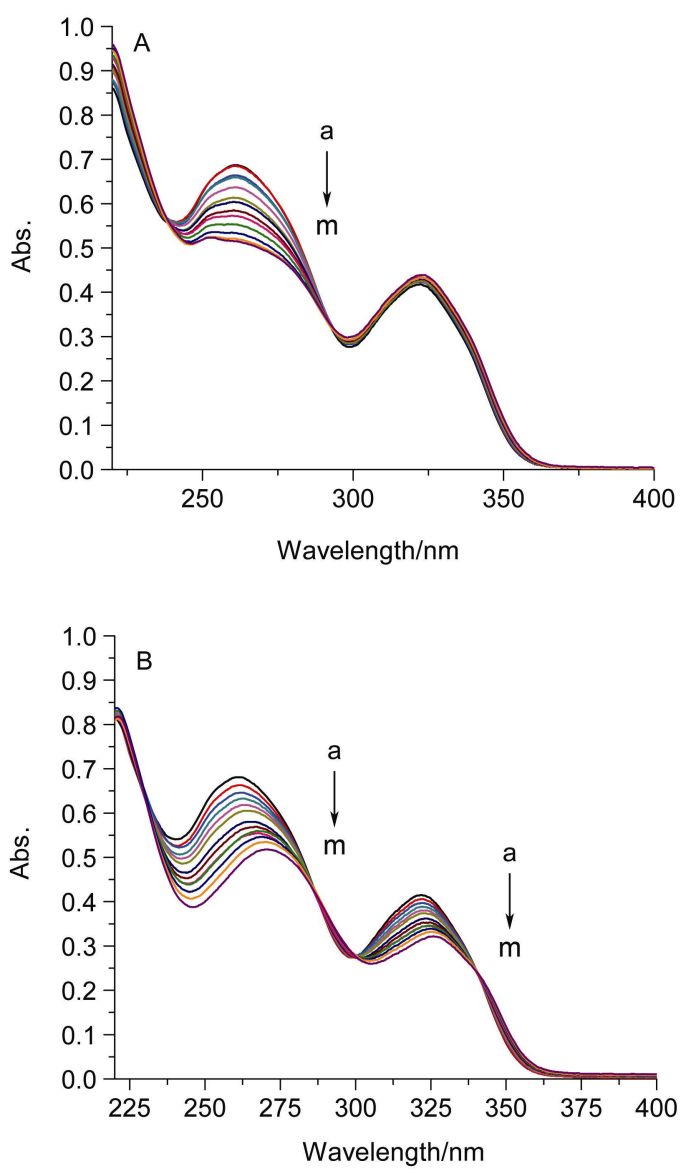

图4 [XDS-6CMV]与 $\mathrm{CB}[n](n=7,8)$ 相互作用的紫外-可见吸 收谱图

Figure 4 UV-Vis absorption spectra of interaction between [XDS-6CMV] and $\mathrm{CB}[n](n=7,8)$

(A) $\mathrm{CB}[7]$, (B) $\mathrm{CB}(8), n[\mathrm{CB}[n](n=7,8)] / n$ ([ XDS-6CMV]): a, $0 ; \mathrm{b}, 0.1$; c, $0.2 ; \mathrm{d}, 0.3 ; \mathrm{e}, 0.4 ; \mathrm{f}, 0.5 ; \mathrm{g}, 0.6 ; \mathrm{h}, 0.7 ; \mathrm{i}, 0.8 ; \mathrm{j}, 0.9 ; \mathrm{k}, 1.0 ; 1,1.1 ; \mathrm{m}, 1.2$ 
1.3.2 [XDS-3CMV]与 $\mathrm{CB}[n](n=7,8)$ 相互作用的 UV-Vis 图谱解析

图 5A, 5B 分别为 $\mathrm{CB}[7], \mathrm{CB}[8]$ 与 $[\mathrm{XDS}-3 \mathrm{CMV}]$ 相互 作用体系的 UV-Vis 图谱. 比较图 4 可以看到, CB[7]与 [XDS-3CMV] 相互作用后的紫外吸收光谱的规律与 [XDS-6CMV]相似，说明[XDS-3CMV]与 $\mathrm{CB}[7]$ 结合时 形成了 $1: 1$ 的包结配合物, 此时紫精单元进入了 CB[7] 的空腔. 计算得到 XDS-3CMV 与 $\mathrm{CB}$ [7]的包结稳定常数 为 $(1.74 \pm 0.31) \times 10^{4} \mathrm{~L} / \mathrm{mol}$. 当 $\mathrm{CB}[8]$ 与 $[\mathrm{XDS}-3 \mathrm{CMV}]$ 相 互作用时, 香豆素单元和紫精单元都受到了瓜环的影 响, 结合 ${ }^{1} \mathrm{H}$ NMR 谱图, 可以推测当 [XDS-3CMV]与 $\mathrm{CB}[8]$ 包结时主要形成聚合物, 在此聚合物中香豆素单 元和紫精单元都进入了 $\mathrm{CB}[8]$ 的空腔(Scheme 2).
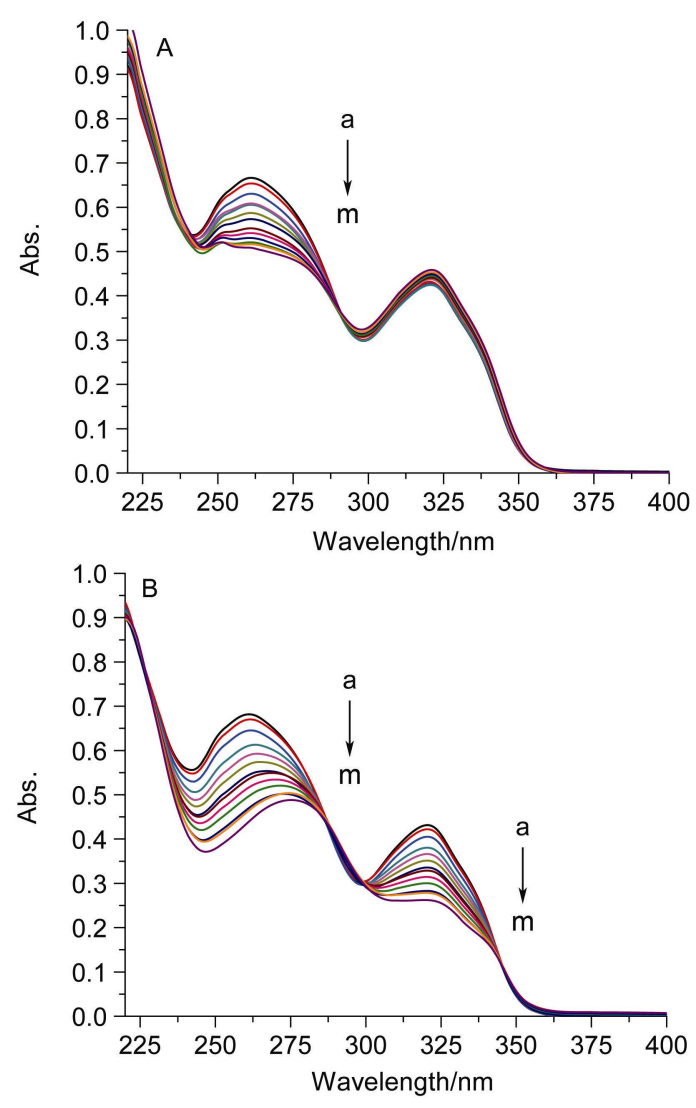

图 5 [ XDS-3CMV]与 $\mathrm{CB}[n](n=7,8)$ 相互作用的紫外-可见 吸收谱图

Figure 5 UV-Vis absorption spectra of interaction between [ XDS-3CMV] and $\mathrm{CB}[n](n=7,8)$

(A) $\mathrm{CB}[7]$, (B) $\mathrm{CB}[8] . n[\mathrm{CB}[n](n=7,8)] / n$ ([ XDS-6CMV]): a , $0 ; \mathrm{b}, 0.1$; c, $0.2 ; \mathrm{d}, 0.3 ; \mathrm{e}, 0.4 ; \mathrm{f}, 0.5 ; \mathrm{g}, 0.6 ; \mathrm{h}, 0.7 ; \mathrm{i}, 0.8 ; \mathrm{j}, 0.9 ; \mathrm{k}, 1.0 ; 1,1.1 ; \mathrm{m}, 1.2$

\section{4 苂光图谱解析}

1.4.1 [XDS-6CMV] 与 $\mathrm{CB}[n](n=7,8)$ 相互作用的荧 光图谱解析

对于具有苂光性质的客体化合物, 荧光光谱可以作 为研究包结物的简易方法. 瓜环没有苂光, 在苂光光谱
上没有特征峰. 设定该体系的激发波长为 $320 \mathrm{~nm}$ ，激发 和发射光谱的狭缝宽度均为 $5 \mathrm{~nm}$. 图 6A, 6B 分别为摩 尔比法测定 CB[7], CB[8]与[XDS-6CMV]相互作用的荧 光图谱, 由于瓜环空腔大小的不同, 我们可以观察图 $6 \mathrm{~A}$ 和 $6 \mathrm{~B}$ 存在很大的区别. 从图 $6 \mathrm{~A}$ 中可以观察到, 随 着 $\mathrm{CB}[7]$ 的加入苂光逐渐减弱. 这是由于缺电子基团紫 精被包进七元瓜环的内部, 影响了整个分子体系的电子 转移和分布, 从而改变了分子的荧光性质. 从图 $6 \mathrm{~B}$ 可 以发现，随着 $\mathrm{CB}[8]$ 的加入，荧光强度明显的下降，这 说明整个客体分子都进入了瓜环内部空腔. 综合以上 ${ }^{1} \mathrm{H}$ NMR, ESI-MS, UV-Vis 以及苂光图谱, 可以推测出 整个分子以 $U$ 型结构进入瓜环空腔. 由于香豆素单元的 刚性平面结构受到了影响，电子分布和外部环境发生变 化，所以分子的苂光强度发生了显著改变. 这也验证了 我们对[XDS-6CMV] 与 $\mathrm{CB}[7], \mathrm{CB}[8]$ 的作用模式的推测 是合理的.

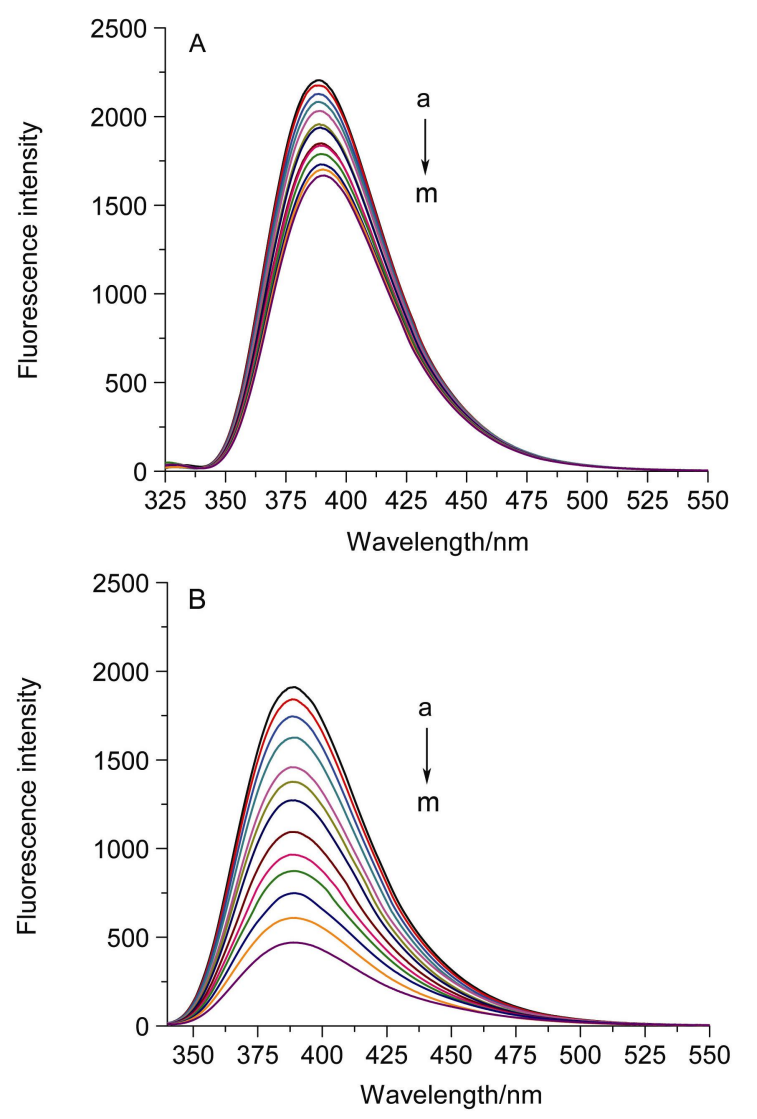

图 6 [XDS-6CMV]与 $\mathrm{CB}[n](n=7,8)$ 相互作用的苂光谱图 Figure 6 Fluorescence spectra of interaction between [XDS$6 \mathrm{CMV}]$ and $\mathrm{CB}[n](n=7,8)$

(A) $\mathrm{CB}[7]$, (B) $\mathrm{CB}(8) . n[\mathrm{CB}[n](n=7,8)] / n$ ([XDS-6CMV]): a, $0 ; \mathrm{b}, 0.1$; c, $0.2 ; \mathrm{d}, 0.3 ; \mathrm{e}, 0.4 ; \mathrm{f}, 0.5 ; \mathrm{g}, 0.6 ; \mathrm{h}, 0.7 ; \mathrm{i}, 0.8 ; \mathrm{j}, 0.9 ; \mathrm{k}, 1.0 ; 1,1.1 ; \mathrm{m}, 1.2$

1.4.2 [XDS-3CMV]与 CB $[n](n=7,8)$ 相互作用的荧 光图谱解析

图 7A, 图 7B 分别是摩尔比法测定的[XDS-3CMV] 
与 $\mathrm{CB}[7], \mathrm{CB}[8]$ 相互作用的苂光图谱. 当[XDS-3CMV] 与 $\mathrm{CB}[7]$ 相互作用时, [XDS-3CMV]的苂光图谱变化不 大. 当 $\mathrm{CB}[8]$ 加入到[XDS-3CMV] 的水溶液时, $390 \mathrm{~nm}$ 处的峰随着 $\mathrm{CB}[8]$ 的加入显著减弱, 而且在 $450 \mathrm{~nm}$ 处形 成了一个新峰，表明[XDS-3CMV] 体系中的刚性结构 及电子分布和转移发生了较大的变化, 因此导致荧光发 生了明显的变化. 这与[XDS-6CMV]和 CB[8]相互作用 时的荧光图谱变化有所不同, 进一步说明 $\mathrm{CB}[8]$ 与 [XDS-3CMV]形成的不是简单的 $1: 1$ 的包结配合物而 是聚合物(Scheme 2).
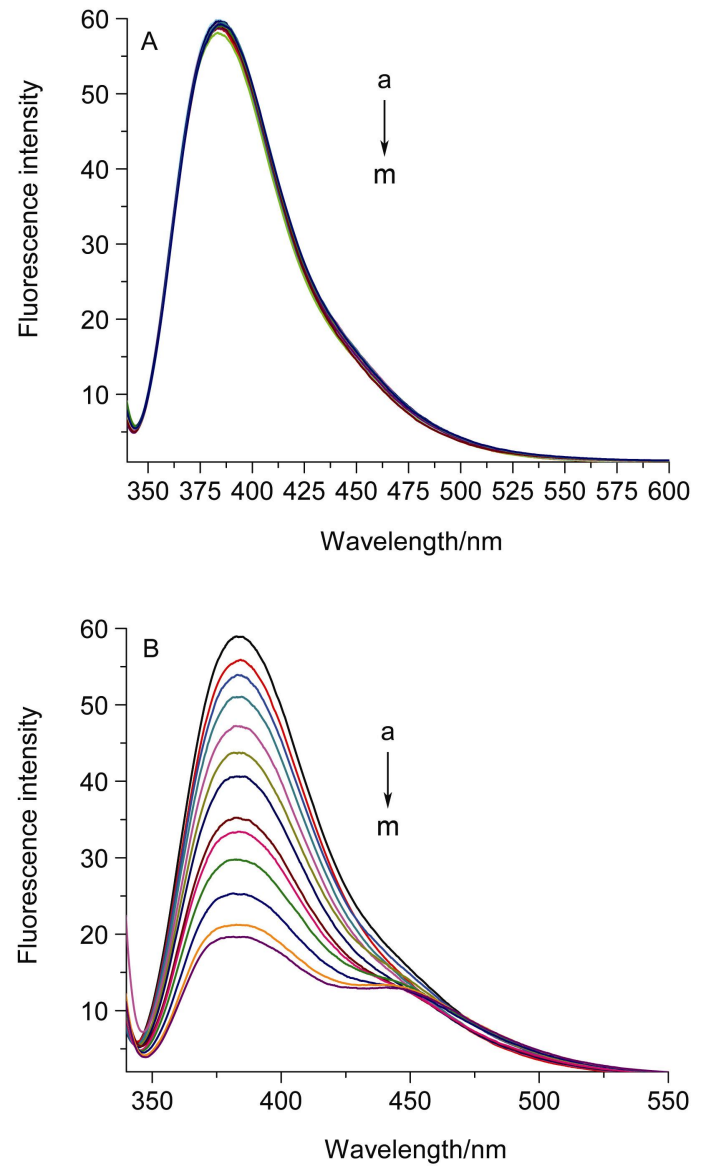

图 7 [XDS-3CMV]与 $\mathrm{CB}[n](n=7,8)$ 相互作用的荧光谱图

Figure 7 Fluorescence spectra of interaction between [XDS$3 \mathrm{CMV}]$ and $\mathrm{CB}[n](n=7,8)$

(A) $\mathrm{CB}[7]$, (B) $\mathrm{CB}(8) . n[\mathrm{CB}[n](n=7,8)] / n$ ([XDS-6CMV]): a, $0 ; \mathrm{b}, 0.1$; c, $0.2 ; \mathrm{d}, 0.3 ; \mathrm{e}, 0.4 ; \mathrm{f}, 0.5 ; \mathrm{g}, 0.6 ; \mathrm{h}, 0.7 ; \mathrm{i}, 0.8 ; \mathrm{j}, 0.9 ; \mathrm{k}, 1.0 ; 1,1.1 ; \mathrm{m}, 1.2$

\section{2 结论}

综合 ${ }^{1} \mathrm{H}$ NMR, ESI-MS, UV-Vis 以及苂光光谱分析 所得到的结果, 说明 [XDS-3CMV], [XDS-6CMV] 与 $\mathrm{CB}$ [7]相互作用时都形成 $1: 1$ 的包结配合物, 此时紫精 单元位于瓜环的空腔内, 其区别仅在于 [XDS-3CMV]$\mathrm{CB}$ [7] 体系中烷基链都处于空腔的外部, 而在 [XDS-6CMV]-CB[7]体系中, 与紫精单元相连的亚甲基
也处于 $\mathrm{CB}[7]$ 的空腔内部. 当 $[\mathrm{XDS}-6 \mathrm{CMV}]$ 与 $\mathrm{CB}[8]$ 相互 作用时, 紫精单元折叠回来和香豆素单元一起呈 $\mathrm{U}$ 型结 构进入 $\mathrm{CB}[8]$ 的疏水空腔形成了更稳定的 $1: 1$ 包结配 合物. [XDS-3CMV]与 $\mathrm{CB}[8]$ 相互作用时，由于碳链长度 不够导致客体分子采取 U 型结构形成 $1: 1$ 包结配合物 不易，因而分子间的紫精单元及香豆素单元共同进入 $\mathrm{CB}[8]$ 空腔形成了超分子聚合物. 这也说明客体分子中 烷基链长度及瓜环尺寸的差异，能直接导致客体分子与 瓜环的包结模式的不同. 目前香豆素衍生物与瓜环相互 作用形成的包结配合物鲜有报道, 本文报道了分子内或 分子间的香豆素单元及紫精单元可以同时进入瓜环的 空腔, 此研究可以为瓜环与香豆素类衍生物在生物学、 药物学以及分析识别等领域的综合研究及潜在应用提 供更加丰富的理论支持.

\section{3 实验部分}

\section{1 仪器与试剂}

试验所用瓜环和[XDS-3CMV], [XDS-6CMV]为本 实验室制备 ${ }^{[28,29]}$, Bruker AVANCE III $400 \mathrm{MHz}$ 超导核 磁共振仪(瑞士 Bruker 公司); Agilent 1100 Series LC/MSD 液一质联用仪(美国 Agilent 公司); UV-2550 紫 外-可见分光光度计(日本 Shimadzu 公司); F-4500 荧光 分光光度计 (日本 Hitcichi High-Technologie 公司); AD-4 Autobalance 电子天平(Perkin-Elmer 公司). 实验用水为 二次蒸馏水. 其他药品和试剂均为市售分析纯.

\section{2 实验方法}

\subsection{1 核磁共振谱的测定}

固定摩尔比法: $\mathrm{D}_{2} \mathrm{O}$ 为溶剂, 以溶剂峰定标, 固定 客体目标化合物 [XDS- $\left.\mathrm{C}_{m} \mathrm{MV}\right](m=3,6)$ 的浓度, 逐渐定 量加入 $\mathrm{CB}[n](n=7,8), 25{ }^{\circ} \mathrm{C}$ 下分别测定不同物质的量 比条件下 $(1: 0,1: 0.5,1: 1)$ 主客体自组装体系的 ${ }^{1} \mathrm{H}$ NMR 图谱.

\subsubsection{ESI-MS 谱图的测定}

按照 $1: 2$ 的物质的量比例称取 [XDS- $\left.\mathrm{C}_{m} \mathrm{MV}\right](m=3$, 6)和 $\mathrm{CB}[n](n=7,8)$, 混合研磨, 超纯水溶解, 超声波助 溶, 测定其 ESI-MS 谱图.

\subsection{3 紫外可见吸收光谱和荧光光谱的测定}

用分析天平精确称取 [XDS- $\left.{ }_{m} \mathrm{MV}\right](m=3,6)$ 置入 $50 \mathrm{~mL}$ 的容量瓶中, 超纯水定容, 超声波助溶, 配制浓 度为 $\left(3 \times 10^{-4} \mathrm{~mol} / \mathrm{L}\right)$ 的水溶液待用. 量取一定量的该溶 液于 $10 \mathrm{~mL}$ 容量瓶中, 再分别量取相同浓度不同体积的 $\mathrm{CB}[n](n=7,8)$ 溶液加入容量瓶中，高纯水定容，配制 主客体物质的量比分别为 $1: 0,1: 0.1,1: 0.2,1: 0.3$, $1: 0.4,1: 0.5,1: 0.6,1: 0.7,1: 0.8,1: 0.9,1: 1.0$, 
$1: 1.1,1: 1.2$ 的一系列溶液. 稳定 $2 \mathrm{~h}$ 后, 分别测定其 紫外可见吸收光谱和苂光光谱.

\section{References}

[1] Kim, J.; Jung, I. S.; Kim, S. Y.; Lee, E.; Kang, J. K.; Sakamoto, S.; Yamaguchi, K.; Kim, K. J. Am. Chem. Soc. 2000, 122, 540.

[2] Miyake, J.; Chujo, Y. Chem. Lett. 2008, 37, 312.

[3] Ko, Y. H.; Kim, E.; Hwang, I.; Kim, K. Chem. Commum. 2007, 1305.

[4] Ong, W.; Gomez-Kaifer, M.; Kaifer, A. E. Org. Lett. 2002, 4, 1791.

[5] Jeon, W. S.; Moon, K.; Park, S. H.; Chun, H.; Ko, Y. H.; Lee, J. Y.; Lee, E. S.; Samal, S.; Selvapalam, N.; Rekharsky, M. V.; Sindelar, V.; Sobransingh, D.; Inoue, Y.; Kaifer, A. E.; Kim, K. J. Am. Chem. Soc. 2005, 127, 12984.

[6] Buschmann, H. J.; Cleve, E.; Jansen, K.; Wego, A.; Schollmeyer, E. J. Incl. Phenom. Macrocycl. Chem. 2001, 40, 117.

[7] Wang, M.; Li, X.; Liu, L. Chin. J. Chem. 2012, 30, 1022.

[8] Wang, G. Q.; G, L.; Du, L. M.; Fu, Y. L. Microchem. J. 2013, 110, 285 .

[9] Li, J. X.; Zhou, L. P.; Luo, Q.; Wang, Y. G.; Zhang, C. Q.; Lu, W.; Xu, J. Y.; Liu, J. Q. Chin. J. Chem. 2012, 30, 208.

[10] Zou, D. P.; Andersson, S.; Zhang, R.; Sun, S. G.; Akermark, B.; Sun, L. C. Chem. Commun. 2007, 4734.

[11] Kim, S. Y.; Jung, I. S.; Lee, E.; Kim, J.; Sakamoto, S.; Yamaguchi, K.; Kim, K. Angew. Chem. 2001, 113, 2177.

[12] Moon, K.; Grindstaff, J.; Sobransingh, D.; Kaifer, A. E. Angew. Chem., Int. Ed. 2004, 43, 5496.

[13] Li, D.-D.; Ren, K. F.; Chang, H.; Wang, H. B.; Wang, J. L.; Chen, C. J.; Ji, J. Langmuir 2013, 29, 14101.

[14] Ma, P. H.; Tao, Z.; Xue, S. F.; Zhu, Q. J.; Wang, S. K.; Yuan, S. W.; Zhang, J. X.; Zhou, X. Chin. J. Org. Chem. 2007, 27, 414 (in
Chinese).

(马培华, 陶 朱, 薛赛凤, 祝黔江, 王仕魁, 袁尚伟, 张建新, 周 欣, 有机化学, 2007, 27, 414.)

[15] Zhang, T. Y.; Sun, S. G.; Peng, X. J.; Tao, B.-B. Chem. J. Chin. Univ. 2012, 33, 292 (in Chinese).

(张同艳, 孙世国, 彭孝军, 陶涁涁, 高等学校化学学报, 2012, 33, 292.)

[16] Moon, K.; Kaifer, A. E. Org. Lett. 2004, 6, 185.

[17] Jeon, W. S., Kim, H. J.; Lee, C.; Kim, K. Chem. Commun. 2002, 1828.

[18] Sun, S. G.; Andersson, S.; Zhang, R.; Sun, L. C. Chem. Commun. 2010, 46, 463.

[19] Jiang, S.; Yang, X. N.; Yang, C. X.; Tong, M. Q.; Zou, D. P.; Wu, Y. J. Tetrahedron. Lett. 2013, 54, 1638.

[20] Chen, H.; Yang, H.; Xu, W. C.; Tan, Y. B. Chin. Chem. Lett. 2013 , $24,857$.

[21] Zou, D. P.; Andersson, S.; Zhang, R.; Sun, S. G.; Akermark, B.; Sun, L. C. J. Org. Chem. 2008, 73, 3775.

[22] Han, Y.; Gu, Y. K.; Xiang, J. F.; Chen, C. F. Chem. Commun. 2012, 48, 11076 .

[23] Han, Y.; Cao, J.; Li, P. F.; Zong, Q. S.; Zhao, J. M.; Guo, J. B.; Xiang, J. F.; Chen, C. F. J. Org. Chem. 2013, 78, 3235.

[24] Chen, C. F. Chem. Commun. 2011, 47, 1674.

[25] Santana, L.; Uriarte, E.; Roleira, F.; Milhazes, N.; Borges, F. Curr. Med. Chem. 2004, 11, 3239.

[26] Yu, D. L.; Suzuki, M.; Xie, L.; Morris-Natschke, S. L.; Lee, K. H. Med. Res. Rev. 2003, 23, 322.

[27] Christie, R. M.; Lui, C. H. Dyes Pigm. 2011, 42, 85.

[28] Cao, X. W.; Lin, W. Y.; Yu, Q. X. J. Org. Chem. 2011, 76, 7423.

[29] Cavar, S.; Kovac, F.; Maksimovic, M. Chem. Food 2009, 117, 135.

[30] Asakura, N.; Hiraishi, T.; Kamachi, T.; Okura, I. J. Mol. Catal. A: Chem. 2001, 174, 1.

(Li, L.; Fan, Y.) 